\title{
Analysis of cyclic phase transformations during additive manufacturing of hardenable tool steel by in-situ X-ray diffraction experiments
}

Jeremy Epp ${ }^{1,2}$, Juan Dong ${ }^{1}$, Heiner Meyer ${ }^{1}$, Annika Bohlen ${ }^{3}$

1: Leibniz Institute for Materials Engineering-IWT Bremen, Badgasteiner Str. 3, 28359, Bremen, Germany

2: MAPEX center for Materials and Processes, Universität Bremen, Bibliothekstr. 1, 28359 Bremen, Germany

3: BIAS - Bremer Institut für angewandte Strahltechnik GmbH, Klagenfurter Str. 5, 28359, Bremen, Germany

Corresponding Author: Jeremy Epp:epp@iwt-bremen.de

\section{Abstract:}

In-situ X-ray diffraction experiments were performed during Laser Metal Deposition of steel X40CrMoV5-1 at the Deutsches Elektronen-Synchrotron (DESY). The measurements were performed with monochromatic high-energy $\mathrm{X}$-ray radiation in transmission, while full diffraction rings were recorded at $10 \mathrm{~Hz}$. The first generated layer is remelted until deposition of the third layer on top, while partial re-austenitizing of the first layer occurs until deposition of the $7^{\text {th }}$ layer. Selftempering effects were analyzed based on martensite tetragonality. Strong decrease of carbon content in solution in martensite occurs during the deposition of the first five layers, resulting in low local hardness.

Keywords: additive manufacturing, laser metal deposition, in-situ X-ray diffraction, tool steel, selftempering

Additive manufacturing ( $A M)$ can be used as a production process for metallic components and is currently one of the rapidly developing advanced manufacturing techniques. Parts are built up layer 
by layer by melting and consolidating the feedstock to generate arbitrary shapes [1]. Energy sources for AM of metallic parts are typically laser or electron beams while powder or wire are used as feedstock. A process which has been used for approximately two decades in industrial application is the laser metal deposition (LMD) [2]. In the past it has mainly been used to repair damaged parts or to enhance surface properties [3] but is nowadays increasingly used to generate complex-shaped parts. Powder is supplied to the building region via nozzles and melted by a laser beam to build a three-dimensional part. For industrial application of AM to build metallic components not only the shape and the porosity of the parts represent critical properties, but the knowledge and the control of the microstructure, residual stresses and distortion are also key issues. This is especially the case for AM parts which need to withstand high thermal and mechanical stresses, like tools for hightemperature applications [4]. FEM simulations are a well-established tool for property prediction in heat treatment or for welding processes and can also be applied for AM processes [5]. They can be used to predict and control microstructure, residual stresses [6] and distortions [7], as well as optimize the process conditions [8] to improve the final mechanical properties. However, in the case of hardenable steels, the multiple layer processing makes the simulations difficult and often unreliable since the starting parameters for each new layer is based on models assuming that the behaviour of already deposited material remains unchanged. In reality, all deposited layers are successively heated up and cooled down with decreasing amplitude for each new layer deposited on their top. In hardenable steels, the rapid cooling after the deposition of one layer will lead to a martensitic microstructure which will be reheated and re-austenitized or even remelted when the second layer is built [8]. With every additional layer, cyclic reheating and cooling will result, leading to modifications of the microstructure. The local material properties of the final built part will consequently depend on the process parameters and on the position within the built sample.

The aim of the present study was therefore to analyze microstructural evolution during the LMD process of hardenable tool steel, in particular the behaviour of already built layers during the 
deposition of the following layers inducing cyclic temperature variations. For this, in-situ X-ray diffraction experiments were performed during real LMD process applied at the Deutsches Elektronen-Synchrotron (DESY) in Hamburg, Germany.

Laser Metal Deposition was used to build steel samples with simple geometry (thin walls). The used steel was the commercially available X40CrMoV5-1 (1.2344). Measurements of chemical composition by optical emission spectroscopy after LMD gave a conform amount of alloying elements, excepted for the carbon content, which was lower than specified (measured value: 0.33 Ma-\%). The particle size distribution of the used powder was between 50 and $100 \mu \mathrm{m}$. The LMD setup was based on a solid state YAG-laser (IPG Photonics) with a wavelength of $1.4 \mu \mathrm{m}$, a power of $300 \mathrm{~W}$ and focal spot size of $0.3 \mathrm{~mm}$ at the working distance of about $5 \mathrm{~cm}$ below the powder delivery nozzles. A 6-axis robot arm was used to ensure a precise movement of the processing head. The process parameters were as follows: Feed rate: 300 m/min; waiting time between each layer: $5 \mathrm{~s}$; height position increment for each layer: $0.3 \mathrm{~mm}$; powder mass flow: $5.6 \mathrm{~g} / \mathrm{min}$. The powder was delivered to the process zone by two nozzles using pressurized Argon flow of 2.4 bar. During the experiments, thin walls with a thickness of about $1.5 \mathrm{~mm}$, a length of $30 \mathrm{~mm}$ and a final height of about $6.5 \mathrm{~mm}$ were built by deposition of 30 successive layers. The thickness of the deposited layer was continuously decreasing during the process, due to inefficient powder delivery and heat accumulation. The deposition was performed on base plates with a size of $20 \times 50 \times 10 \mathrm{~mm}^{3}$ from the same material as the powder, clamped on a massive copper holder for heat dissipation.

The diffraction measurements were performed on Beamline P07-EH3 at DESY with high energy monochromatic X-ray beam $(97.6 \mathrm{keV})$ in transmission geometry, through the complete thickness of the built walls. The beam size was defined by slits to a height of $200 \mu \mathrm{m}$ and a width of $1 \mathrm{~mm}$, in order to ensure good spatial resolution together with sufficient grain statistic and high diffraction signal intensity. The experimental set-up and the principle of the measurement configuration are presented in Figure 1. 

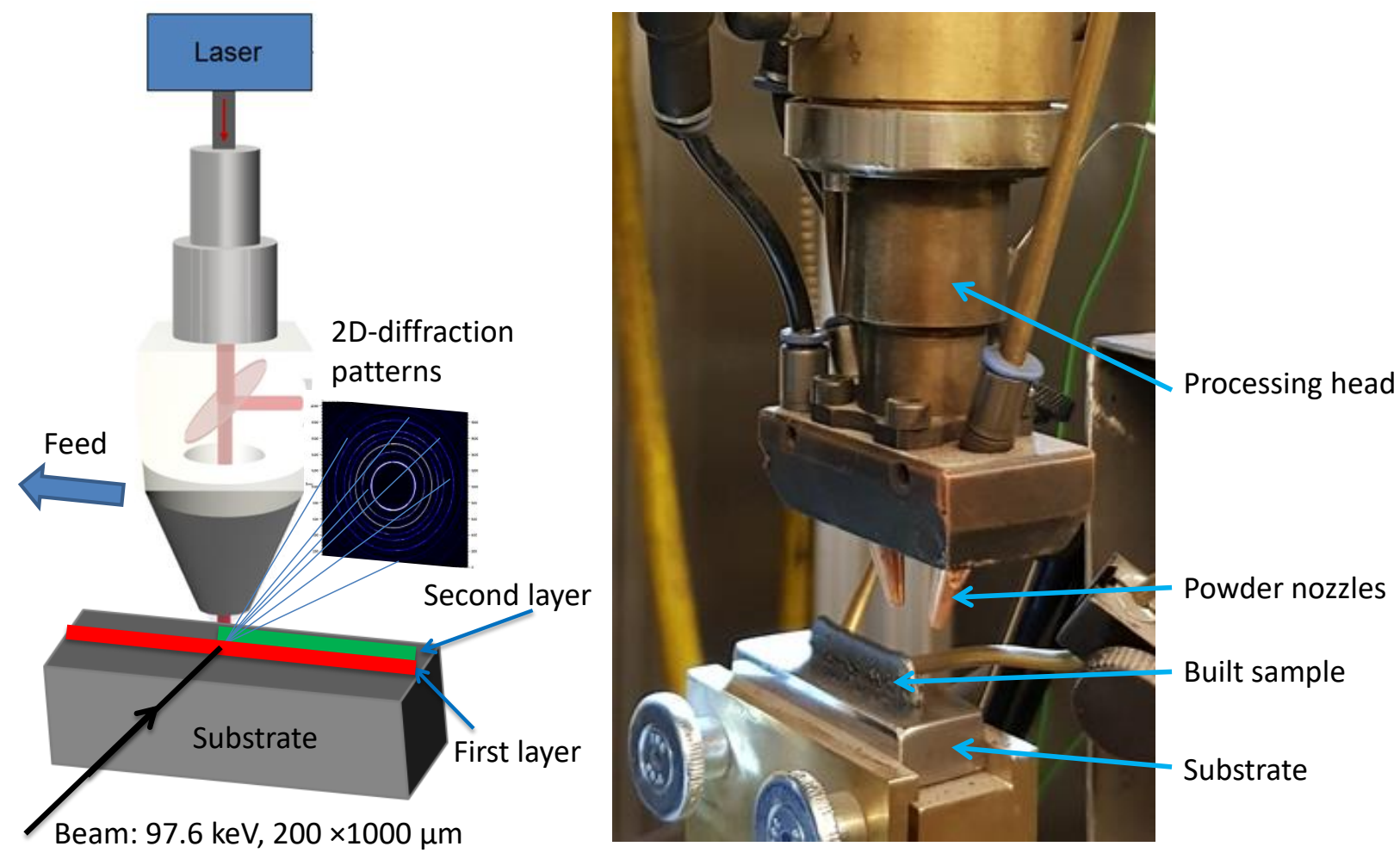

Figure 1: Experimental set-up for the in-situ XRD experiments during LMD: a) sketch of the set-up; b) Picture of the $L M D$ processing head and of a built sample

In one single experiment, the beam was kept at fixed position in the first built layer, directly above the substrate during the whole LMD process. With this strategy, it was possible to achieve a reasonable time resolution by using a 2D detector (Perkin-Elmer with $2048 \times 2048$ pixels and a pixel size of $200 \mu \mathrm{m}$ ) with collection time of $0.1 \mathrm{~s} /$ frame. Full diffraction rings were measured in the range of 0 to $8^{\circ} 2 \theta$ positioned at a distance of $1.42 \mathrm{~m}$ behind the process zone. During the layer deposition, heating rates up to $30000 \mathrm{~K} / \mathrm{s}$ or more occur while during subsequent cooling, cooling rates between 300 and $2000 \mathrm{~K} / \mathrm{s}$ are reached in the temperature range above $1000{ }^{\circ} \mathrm{C}$. During further cooling, the cooling rate decreases continuously to reach low values below $20 \mathrm{~K} / \mathrm{s}$. As a consequence the time resolution of the measurements only allow a rough evaluation of the transformations and their kinetics during heating and during the first stages of cooling from maximum temperature, in particular during deposition of the first layers. However, it was possible to analyze the microstructural evolutions with reasonable time resolution and high data quality during cooling in 
the temperature range below $1000{ }^{\circ} \mathrm{C}$. Through the use of a two-color pyrometer, temperature information was obtained from the process zone with a time resolution of $1 \mathrm{~ms}$, while a thermocouple was positioned at the surface of the substrate (time resolution: $10 \mathrm{~ms}$ ).

For each experiment, up to 5500 single 2D diffraction frame were recorded. The 2D patterns were azimuthally integrated over an angular range of $20^{\circ}$ using the fast PyFAl software of ESRF [9] and further analyzed as conventional intensity vs. $2 \theta$ or q (scattering vector) patterns. Figure 2 shows a sequence of integrated diffraction patterns, recorded in the first layer during the generation and subsequent fast cooling of the second layer on top.

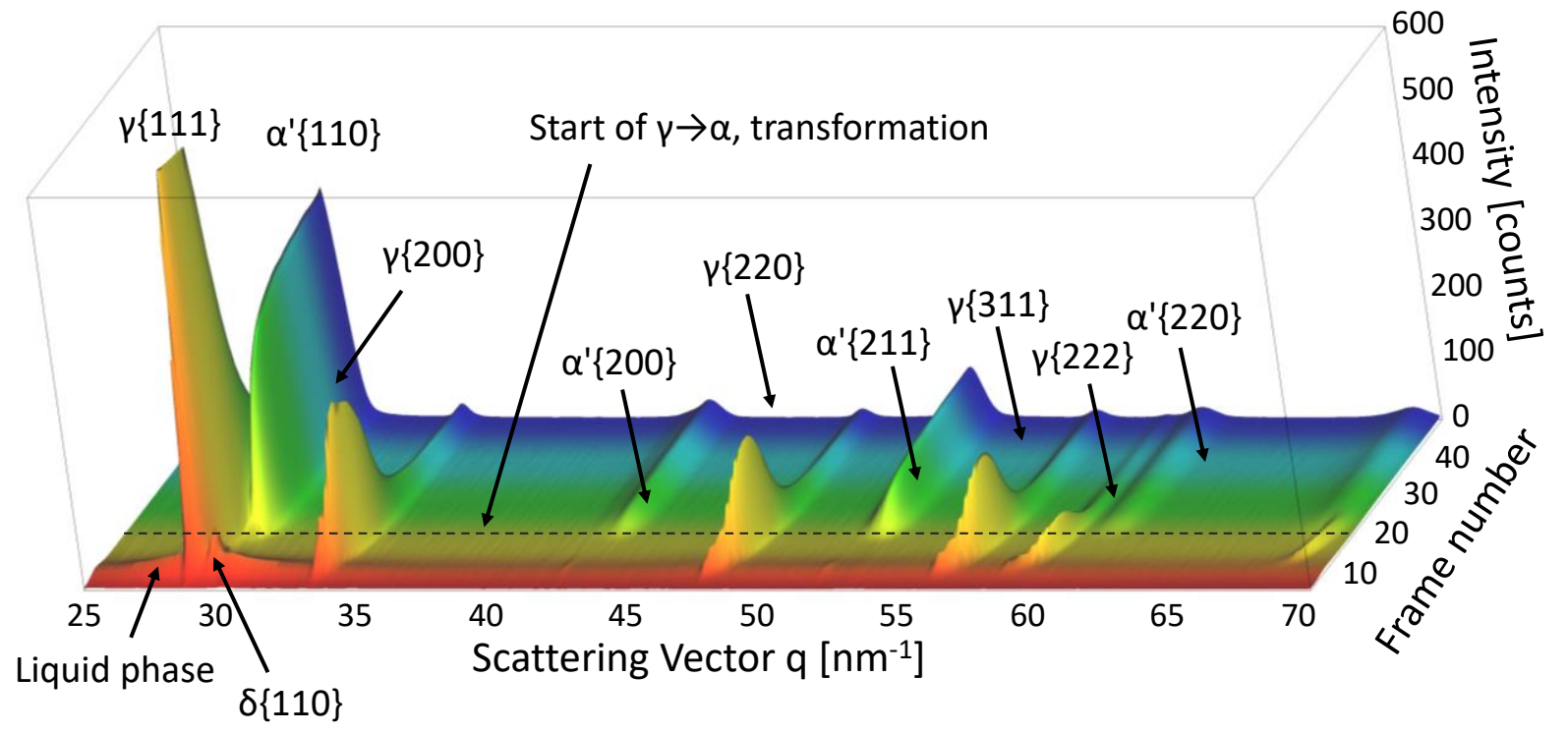

Figure 2: 3D view of 45 integrated diffraction patterns recorded in the first layer during generation and cooling of the second built layer on top during the LMD process

First, the liquid phase can be identified in the form of very broad low-intensity peaks in the first 3 frames, followed by increasing peaks of $\delta$-ferrite (BCC), together with austenitic peaks (FCC). The peaks of $\delta$-ferrite rapidly disappear (within few measurements) and the austenite peaks grow strongly. After only 12 frames (about $1.2 \mathrm{~s}$ after deposition of the layer) austenite $\rightarrow$ martensite (BCT) transformation starts and is ongoing during the whole subsequent cooling period. A certain amount of austenite remains present in the microstructure. The diffraction patterns recorded 
during subsequent deposition of the next layers on top, show that a full remelting of the first layer takes place and the observed transformation sequence is repeated.

For precise and detailed analysis of the sequence of phase transformations and further microstructural evolution in particular tempering effects, the integrated diffraction patterns were analyzed using the convolution-based Rietveld refinement implemented in TOPAS 4.2 (Bruker-AXS) [10]. In this approach, the observed diffraction peaks are modelled by convolution of various functions that represent the instrumental and sample contributions to the peaks profiles. The calibration parameters and the instrumental contribution to the diffraction peak profiles were obtained using measurements of NIST Standard $\mathrm{LaB}_{6}$ powder. For first approximation and fast fitting of all measurements within one experiment, a simplified microstructural model was used, where only austenite and martensite were taken into account. Liquid phase, delta ferrite and possible precipitates/ carbides were neglected.
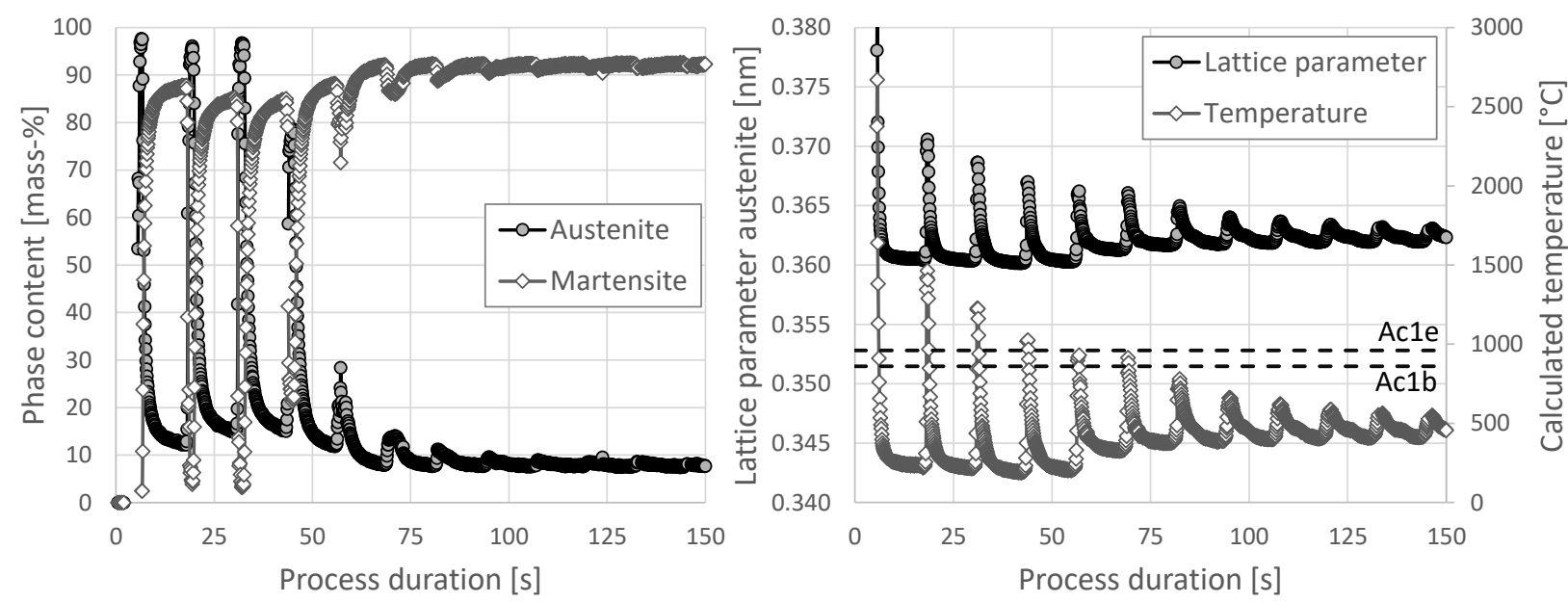

Figure 3: Evolution of phase contents (a) as well as austenite lattice parameter and calculated temperature (b) in the first built layer during its generation and subsequent deposition of the next 11 layers on top

Figure 3a shows the time-resolved evolution of the austenite and martensite contents at a fixed position in the first built layer during its generation and deposition of the next 11 layers built on top. Further, in order to evaluate the temperature within the investigated volume, an approach using 
the determined lattice parameter of austenite and an evaluation based on the model given by Onink et al. was considered [11]. For this, the nominal carbon content of 0.33 Mass- $\%$ as constant value and a thermal expansion coefficient of $22.5 \times 10^{-6} \mathrm{~K}^{-1}$ determined from dilatometric curves were used as first approximation. The temperatures Ac1b and Ac1e for beginning and completed austenitizing determined from dilatometric experiments with heating rates of $1000 \mathrm{~K} / \mathrm{s}$ are indicated in Figure 3b. With increasing number of subsequent layers on top, the amplitude of the thermal cycles decreases. The remelting of the first layer takes place until the deposition of the third layer, leading to a full decomposition of the built martensite and the generation of new virgin martensite during the subsequent cooling period. After the deposition of the third layer, only partial re-austenitizing of the first layer takes place until layer 7 . This is due to the periodic decrease of the maximum temperature induced in the first layer with every new layer on top. These results agree well with the calculated temperature, showing that the austenite formation temperature is not reached anymore after the deposition of the $7^{\text {th }}$ layer. Beyond this process stage, the maximum temperature reached in the first layer is too low to induce a martensite $\rightarrow$ austenite transformation. However, during the remaining process a periodic temperature increase and decrease can still be observed in layer 1 with decreasing amplitude, while the mean temperature increases continuously during the whole process. Data from thermocouples placed on the substrate showed the same behaviour with an overall temperature increases during the process due to heat accumulation in the substrate and in the clamping device.

The resulting time-temperature cycles in the first layer are expected to induce microstructural changes, in particular regarding self-tempering effects of martensite. Therefore, the evolution of the BCT martensite lattice parameters " $c$ " and "a" obtained from the Rietveld refinements were evaluated in detail during the whole process. The martensite tetragonality ratio "c/a" is known to be linearly dependent on the carbon content in solid solution in martensite as reported and summarized by Cheng et al. based on data from several studies [12]. This dependence can therefore 
be used to determine the carbon content in solution in martensite by the following equation: $\% \mathrm{C}=((\mathrm{c} / \mathrm{a})-1) / 0.0443$, with $\% \mathrm{C}$ in Mass-\% [13]. One main advantage of using this method, is that no information about the temperature is required for precise calculation of the carbon content, since both lattice parameters are used and therefore effects of thermal expansion are self-correcting, as already demonstrated for in-situ experiments during quenching of low alloy steel [13].

The evolution of tetragonality and resulting carbon content in solution in martensite during the LMD process is shown in Figure 4. As for the results presents in Figure 3, the plotted data were recorded at fixed position in the first layer during its generation and subsequent deposition of the next 11 layers on top. The results show that during the cooling of the first layer, the calculated carbon content in the formed martensite increases to reach maximum value around 0.36 Mass-\%, just before the deposition of the second layer. This value corresponds well to the nominal chemical composition of the investigated steel, which confirms that the employed method to estimate the carbon content in solution gives reliable results and additionally that the first built martensite during cooling of the first layer contains the full amount of carbon of the steel. During deposition of the second layer and full remelting of the first layer, the martensite is fully retransformed into austenite during heating, which then transforms again into martensite during further cooling. However, the determined carbon content of the built martensite reached only 0.315 Mass-\% which is below the original value of 0.36 Mass-\%. After deposition of the third layer (still inducing remelting and fully retransformation in austenite of the first layer), the maximum carbon content of the formed martensite reached a slightly lower value of 0.30 Mass-\%. After deposition of layer 4 , without remelting and with only partial reaustenitizing of the first layer, the average carbon content of the remaining and of the new built martensite reaches a maximum value of $0.23 \mathrm{Mass}-\%$. During the generation of the $5^{\text {th }}$ layer, the carbon content in martensite drops to a value of about 0.15 Mass\%. During deposition of the following layers, only small changes can be observed and the carbon content in solution remains in the range of 0.15 Mass-\%. 
These results demonstrate that the remelting of the first layer during deposition of the second and third layers on top leads to a depletion of carbon in the matrix, which is expected to be due to the generation of precipitates (in particular carbides), as already observed in other steels [8]. During the deposition of the subsequent layers with only partial re-austenitizing of the first layer, the remaining martensite is losing carbon due to the tempering effects as a consequence of the elevated temperature [14], while the new formed martensite only slightly increases the average carbon content in solution. The elevated temperature generated during deposition of the following layers on top leads to further tempering effects, finally resulting in a state with strongly decreased carbon content. After deposition of the $7^{\text {th }}$ layer this state is remaining stable until the end of the process and is indicating that the existing temperature field does not induce further significant microstructural evolution.

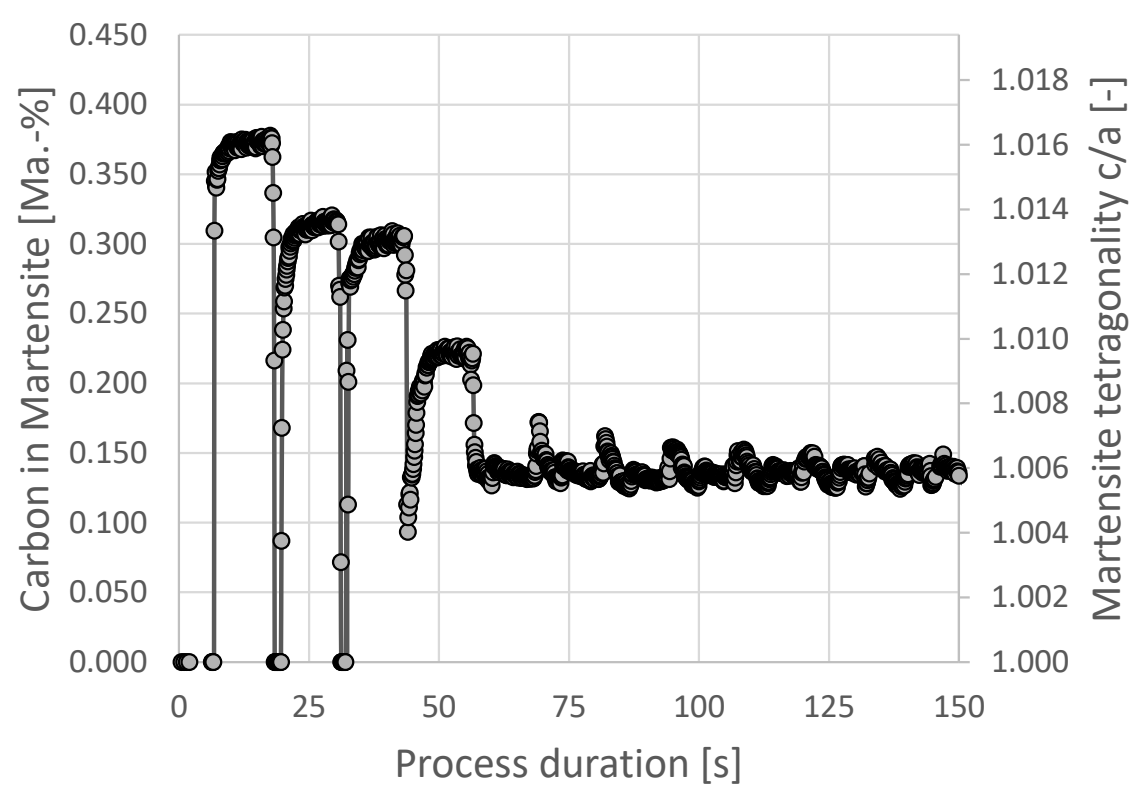

Figure 4: Evolution of carbon content in solution in martensite calculated from tetragonality ratio in the first built layer during its generation and subsequent deposition of the next 11 layers on top Comparable evaluation of in-situ measurements performed at different height positions of walls built with the same process parameters showed that maximum self-tempering effects are induced at about $2 / 3$ of the total height of the wall and the resulting hardness measured after the process 
decreases from 500 HV1 directly above the substrate to lowest values of $350 \mathrm{HV} 1$ at the position of maximum self-tempering. In the top region of the wall, full re-austenitizing took place until deposition of the last layer leading to virgin martensite with high hardness of 700 HV1.

These experimental results give first insights into ongoing microstructural evolution during additive manufacturing of hardenable steel by laser metal deposition and give quantitative information about local multiple phase transformations and self-tempering effects occurring during the process. Further evaluations are currently ongoing to determine precisely the local behaviour at different height positions additionally taking into account precipitate formation, which has already been confirmed qualitatively.

Acknowledgements:

This IGF Project AIF $19308 \mathrm{~N}$ of the FOSTA was supported via AiF within the program for promoting the Industrial Collective Research (IGF) of the German Ministry of Economic Affairs and Energy (BMWi), based on a resolution of the German Parliament. The authors gratefully acknowledge Dr. Florian Wagner, Niels Prager and Rik Fisser from Gall und Seitz GmbH for providing the LMD equipment. We also acknowledge DESY (Hamburg, Germany), a member of the Helmholtz Association HGF, as well as Helmholtz-Zentrum Geesthacht and in particular Dr. Torben Fischer for assistance during the experiments and for the provision of experimental facilities at Petra III.

[1] D. D. Gu, W. Meiners, K. Wissenbach, R. Poprawe, International Materials Reviews, 57,3 (2012) 133

[2] E. M. Birger, G. V. Moskvitin, A. N. Polyakov, V. E. Arkhipov, Welding International 25 (2011) 234

[3] C. Atwood, M. Ensz, D. Greene, M. Griffith, L. Harwell, D. Reckaway, T. Romero, E. Schlienger, J. Smugeresky, in: E. Beyer (Ed.), Proceedings of ICALEO'98, Orlando, FI., 1, 1998

[4] F. Klocke, K. Arntz, M. Teli, K. Winands, M. Wegener, S. Oliari, Procedia CIRP 63 (2017) 58 
[5] T. Amine, J.W. Newkirk, F. Liou, Case Studies in Thermal Engineering 3 (2014) 21

[6] H. Köhler, R. Rajput, P. Khazan, J. Rebelo Kornmeier, 8th Int. Conf. on Laser Assisted Net Shape Engineering (LANE 2014), Physics Procedia 56, Elsevier B.V. Amsterdam, 250 (2014)

[7] L. Papadakis, C. Hauser, Prod. Eng. Res. Devel. 11 (2017) 389

[8] N.S. Bailey, C. Katinas, Y.C. Shin, Journal of Materials Processing Technology 247 (2017) 223

[9] G. Ashiotis, A. Deschildre, Z. Nawaz, J. P. Wright, D. Karkoulis, F. E. Picca, J. Kieffer, Journal of applied crystallography, 48,2 (2015) 510

[10] A. A. Coelho, Journal of Applied Crystallography, 51,1 (2018) 210

[11] M. Onink, C. M. Brakman, F. D. Tichelaar, E. J. Mittemeijer, S. van der Zwaaag, Acta Metallurgica et Materialia, 29 (1993) 1011

[12] L. Cheng, A. Böttger, T. H. de Keijser, E. J. Mittemeijer, Scripta Metall et Mater 24 (1990) 509

[13] J. Epp, T. Hirsch, C. Curfs, Metallurgical and Materials Transaction A, 43 (2012) 2210

[14] J. Epp, Materials Research Proceedings 2 (2017) 283 
Supplementary file: Evaluation of 2D-diffraction patterns at different process steps and

\section{temperature evolution}

For each experiment, up to 5500 single 2D diffraction frame were recorded. The 2D patterns were azimuthally integrated over an angular range of $20^{\circ}$ using the fast PyFAI software of ESRF and further analysed as conventional intensity vs. $2 \theta$ or q (scattering vector) patterns, using the Rietveld method.

In Figure 1, a view of typical diffraction data collected at about $200{ }^{\circ} \mathrm{C}$ is presented as 2D-pattern with the position of the rings of the different hkl-planes of martensite (BCT) and austenite (FCC). The azimuthally integrated region of $20^{\circ}$ is marked, as well as the scattering vector. The corresponding diffraction pattern after azimuthal integration is shown below in Figure 2, where the peaks of both phases can be observed.

A sequence of four datasets recorded during the process are shown in Figure 3. The 2D raw data is shown on the left side, while the integrated diffraction patterns are presented beside. Four characteristic process steps are presented: the liquid phase at maximum temperature, represented by the diffuse rings leading to very broad low-intensity peaks (a); the fully austenitic region during subsequent cooling (b); the beginning of martensitic transformation at slight undercooling below Martensite start, where high amount of austenite is still present (c); pattern recorded at minimum temperature between two layer depositions where high amount of martensite is built and about 30 Mass-\% of remaining austenite $(\mathrm{d})$.

Figure 4 presents four pictures taken at several steps during the process: a) beginning of the process; b) after deposition of the first layer; c) at intermediate process step; d) after deposition of the last layer during final cooling. In particular in the last picture, the heat propagation from the left to the right side of the sample (in scanning direction) allows an estimation of the heat accumulation during the process. 


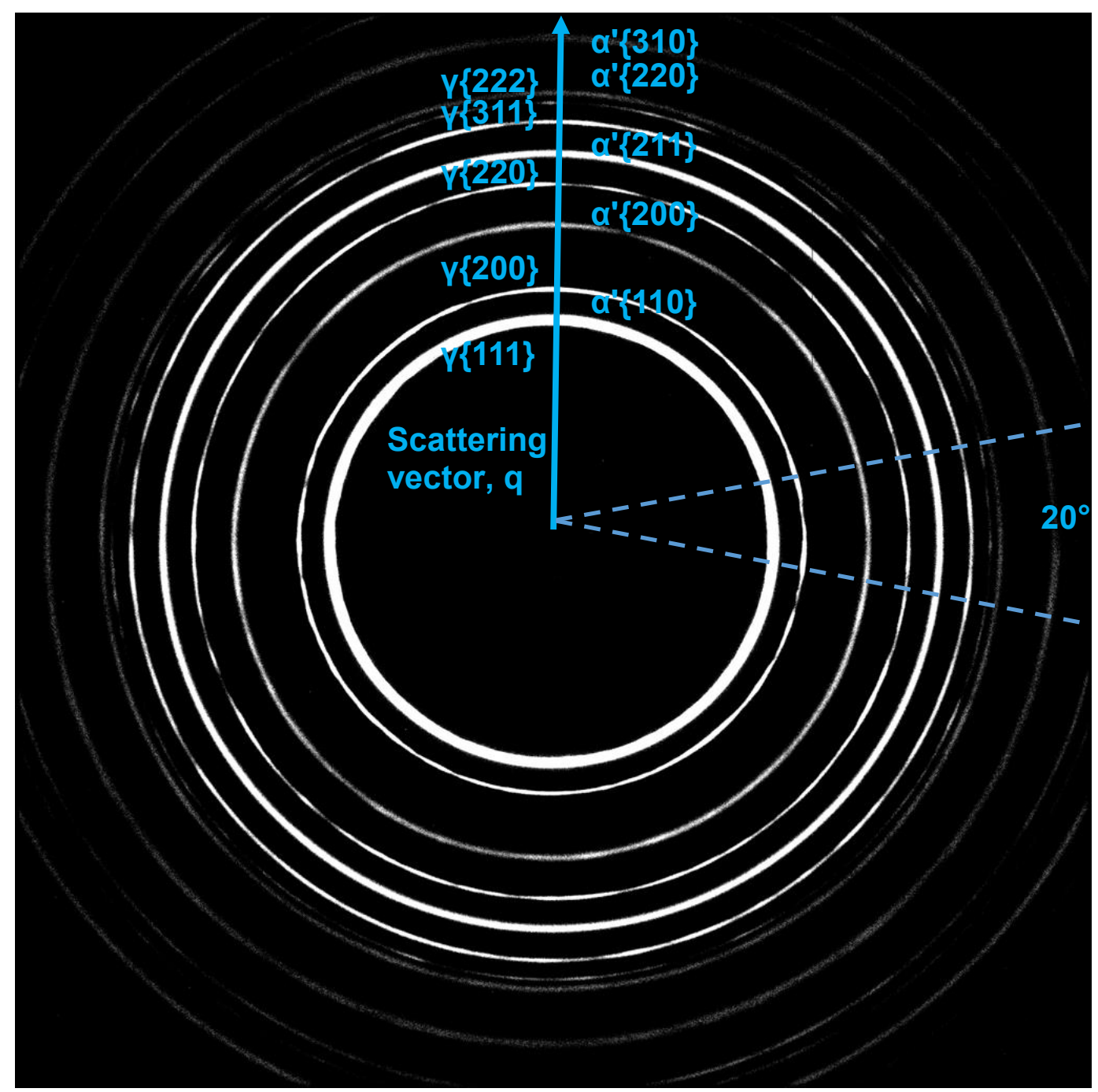

Figure 1: 2D-Diffraction pattern collected at about $200{ }^{\circ} \mathrm{C}$ during cooling

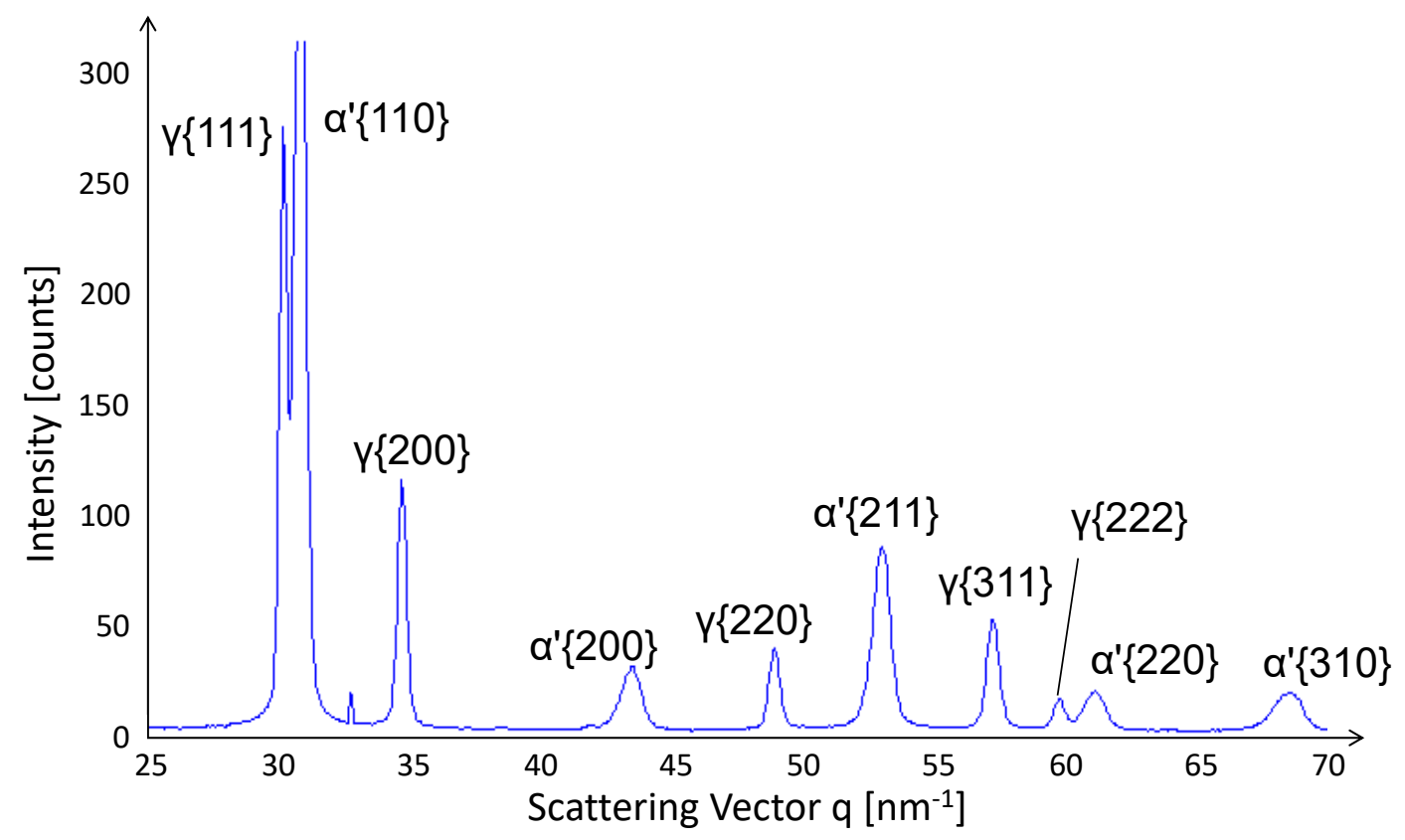

Figure 2: Integrated diffraction pattern of Figure 1 with intensity as a function of the scattering vector 

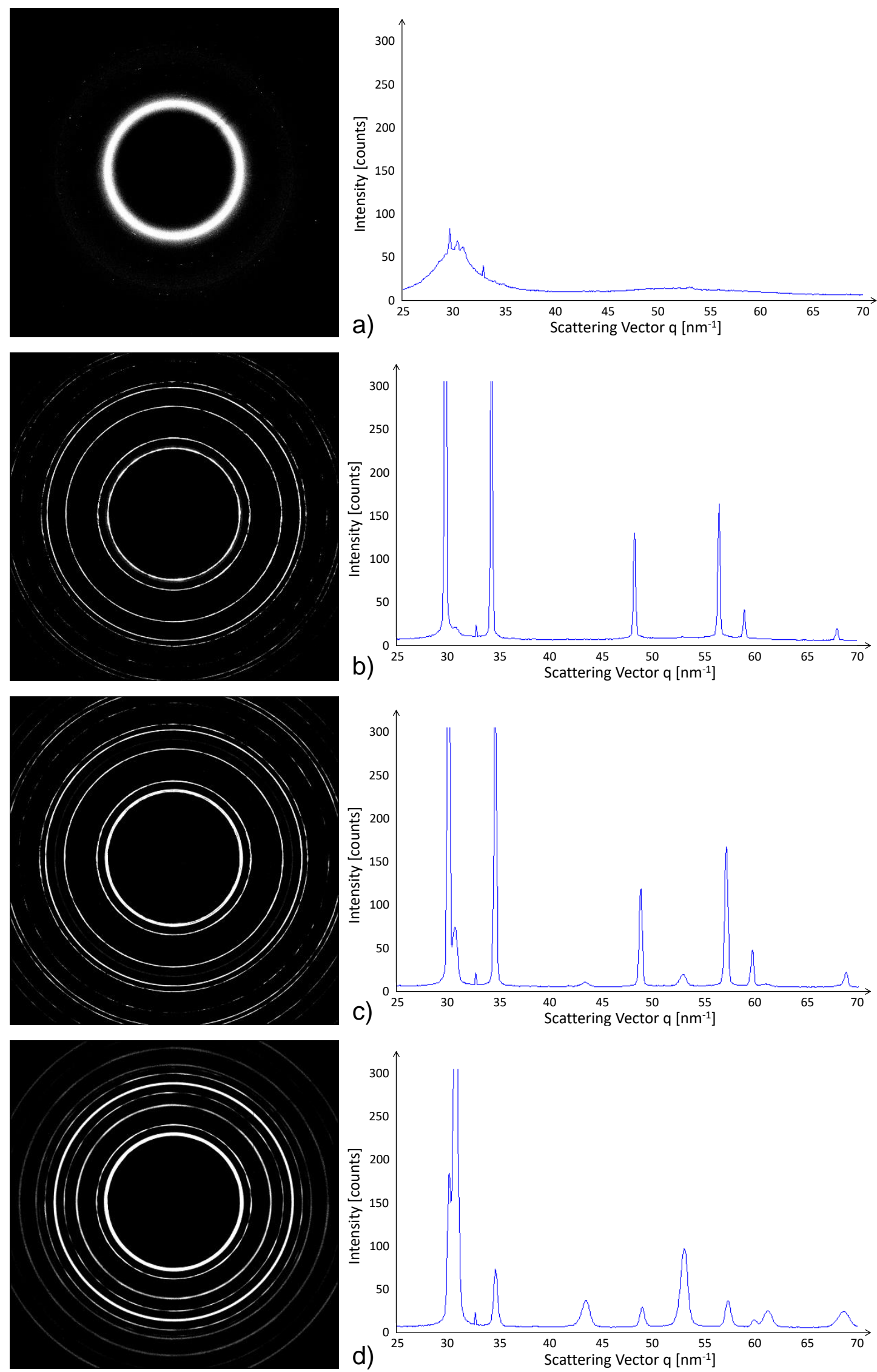

Figure 3: Sequence of 2D- and integrated diffraction patterns recorded during the process at different steps: a) liquid phase at highest temperature; b) fully austenitic region during cooling; $c$ ) beginning of martensitic transformation; d) lowest temperature between two layer depositions 


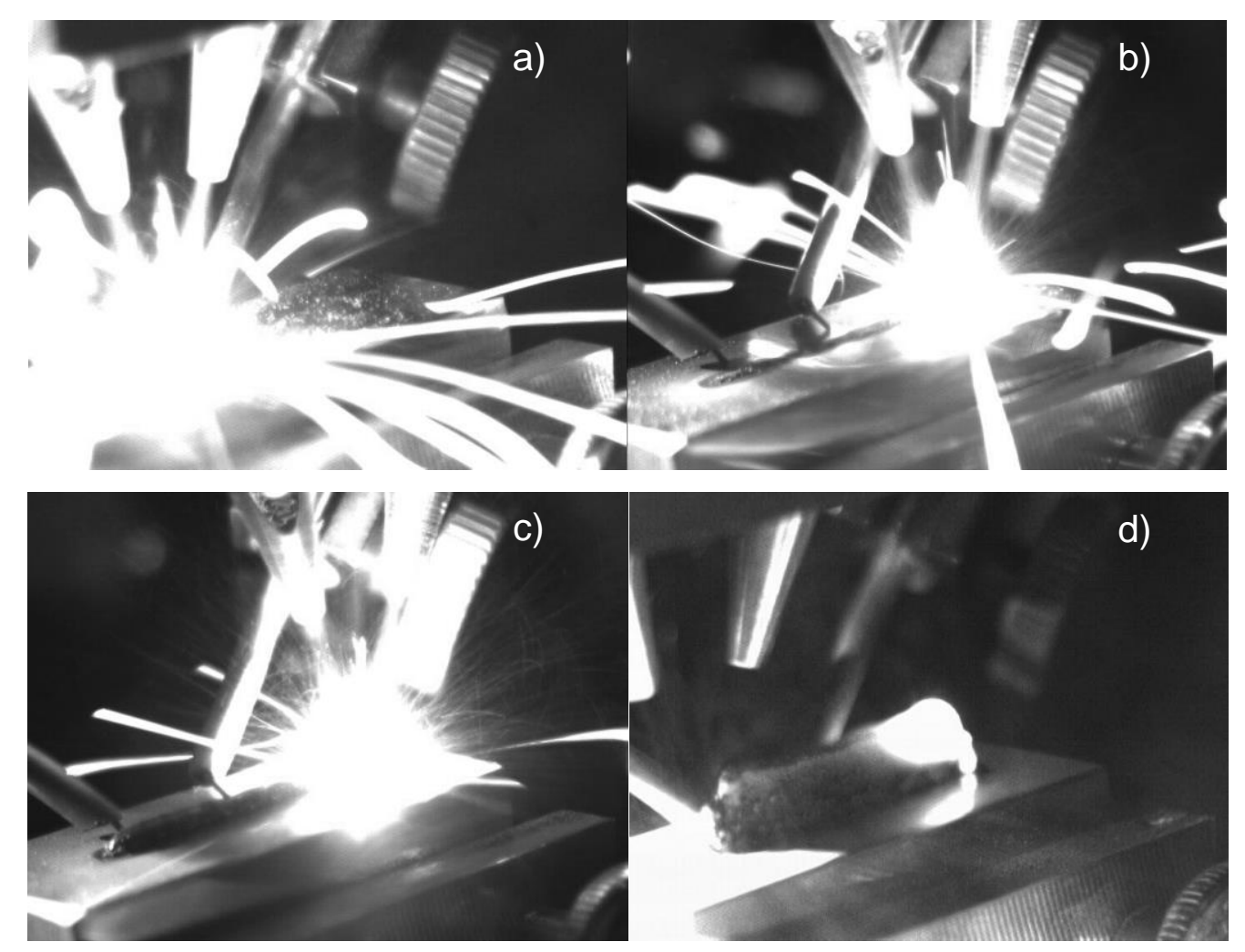

Figure 4: Pictures of the built sample at several steps of the process

The temperature evolution during the process is displayed in Figure 5 as a comparison between the pyrometer data, the thermocouple data measured on the substrate and the calculated temperature based on the determined austenite lattice parameter together with the model as described in the manuscript. It can be seen that the agreement between the pyrometer data and the calculated values is very good, in particular during deposition of the first layers. Peak temperatures measured by the pyrometer are about 150 to $200^{\circ} \mathrm{C}$ higher than the calculated data. This can be attributed to the lower time resolution of the diffraction data $(10 \mathrm{~Hz}$, compared to $1000 \mathrm{~Hz}$ for the pyrometer), which does not allow to detect these peak temperatures. After deposition of the $5^{\text {th }}$ layer, increasing discrepancies between the pyrometer data and the calculated temperatures can be noticed. This can be attributed to the fact that the pyrometer still measures the surface temperature of every new deposited layer, while the synchrotron data were measured through the volume of the first built layer, where much lower temperatures are generated. A comparison with the thermocouple data is therefore more indicated for the further process steps. There, the temperature amplitude is much lower due to the higher distance to the process region. Nevertheless, the measured temperature profile is very similar to the calculated temperature and the overall increase due to heat accumulation through the process can be distinguished in both data sets. 


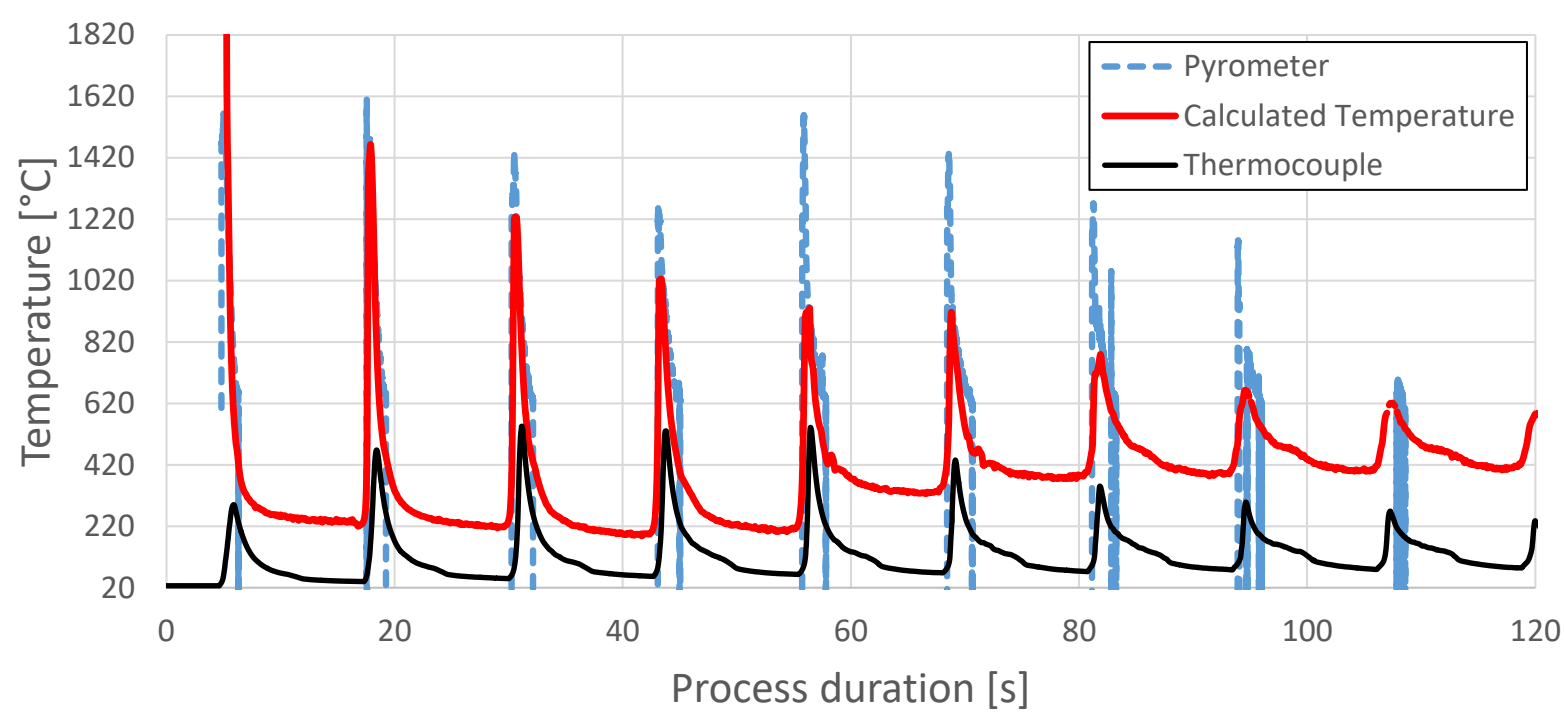

Figure 5: Temperature evolution recorded by the pyrometer and by the thermocouple compared to the calculated temperature based on the measured austenitic lattice parameter

\section{Acceptance letter:}

$\begin{array}{ll}\text { Date: } & \text { Sep 14, } 2019 \\ \text { To: } & \text { "Jérémy Epp" epp@iwt-bremen.de } \\ \text { cc: } & \text {;dong@iwt-bremen.de;hmeyer@iwt-bremen.de; bohlen@bias.de } \\ \text { From: } & \text { Sudarsanam Babu eesserver@eesmail.elsevier.com } \\ \text { Reply To: } & \text { Sudarsanam Babu sbabu@utk.edu } \\ \text { Subject: } & \text { Your Scripta Materialia Submission SMM-19-1352R1 }\end{array}$

Dear Dr Epp,

We are pleased to inform you that your manuscript entitled Analysis of cyclic phase transformations during additive manufacturing of hardenable tool steel by in-situ X-ray diffraction experiments, editorial reference number: SMM-19-1352R1 has been accepted for publication in Scripta Materialia.

Your accepted manuscript will now be transferred to our production department and work will begin on creation of the proof. If we need any additional information to create the proof, we will let you know. If not, you will be contacted again in the next few days with a request to approve the proof and to complete a number of online forms that are required for publication.

We thank you for selecting Scripta Materialia for publishing your paper.

Sincerely,

Kazuhiro Hono, Ph.D.

Editor

Scripta Materialia 